\title{
Reseau Social Et Inadequation «Competence-Emploi) Dans Le Processus D'insertion Des Diplomes Du Brevet De Technicien Superieur (Bts) En Cote d'Ivoire : Cas des étudiants des établissements Loko, Pigier, et Inprat
}

\author{
Akpe Yapo Hermann \\ Enseignant-Chercheur, Institut des sciences anthropologiques de \\ développement (ISAD), Université Félix Houphouët-Boigny de Cocody, \\ Abidjan, Côte d'Ivoire \\ Yapo Lucas Delmas \\ Enseignant-chercheur, Institut des sciences anthropologiques de \\ développement (ISAD), Université Félix Houphouët-Boigny de Cocody, \\ Abidjan, Côte d'Ivoire \\ Kouassi Aya Sainte Sébastienne \\ Attaché de Recherche, Centre Ivoirien de Recherches Economiques et \\ Sociales, Université Félix Houphouët-Boigny de Cocody, \\ Abidjan, Côte d'Ivoire
}

URL:http://dx.doi.org/10.19044/esj.2019.v15n4p174

\section{Résumé}

La littérature scientifique désigne le capital social ou réseau social comme un dispositif informel de référence privilégié dans le processus d'insertion des diplômés de l'enseignement supérieur. La présente étude se consacre entièrement à la validation d'une hypothèse. Celle qui présume que « le recours au réseau social dans le processus d'insertion est la cause de la non-conformité ou de l'inadéquation "compétence-emploi) rencontrées par les diplômés sur le marché de l'emploi. Pour y parvenir, un échantillon de 276 diplômés a été interrogé par questionnaire sur l'appréciation de leur formation et sur l'utilité du réseau social. Il leur a été demandé également leurs Opinions concernant la conformité «compétence-emploi » et sur connaissances des compétences exigées par le marché de l'emploi. En guise d'information complémentaire, un guide d'entretien administré à 24 diplômés en situation emploi. Les résultats montrent que le recours au réseau social, dans le processus d'insertion, ne garantit pas la conformité ou l'adéquation «compétence-emploi) chez les diplômés sur le marché de l'emploi. 
Mots clés : compétence, employabilité, diplôme, formation professionnelle

\title{
Social Network and Inadequacy between "Competence-Employment" in the Insertion Process of Holders of Senior Technician Certificate in Ivory Coast: Case of the Students of the Establishments of Loko, Pigier, and Inprat
}

\author{
Akpe Yapo Hermann \\ Enseignant-Chercheur, Institut des sciences anthropologiques de \\ développement (ISAD), Université Félix Houphouët-Boigny de Cocody, \\ Abidjan, Côte d'Ivoire \\ Yapo Lucas Delmas \\ Enseignant-chercheur, Institut des sciences anthropologiques de \\ développement (ISAD), Université Félix Houphouët-Boigny de Cocody, \\ Abidjan, Côte d'Ivoire \\ Kouassi Aya Sainte Sébastienne \\ Attaché de Recherche, Centre Ivoirien de Recherches Economiques et \\ Sociales, Université Félix Houphouët-Boigny de Cocody, \\ Abidjan, Côte d'Ivoire
}

\begin{abstract}
The scientific literature refers to social capital or social network as a privileged informal reference device in the process of integration of graduates of higher education. This study is entirely devoted to the validation of a hypothesis. That which assumes that "the use of the social network in the process of insertion is the cause of the non-conformity or the inadequacy" competence-employment) met by the graduates on the labor market. To achieve this, a sample of 276 graduates was interviewed by questionnaire on the assessment of their training and the usefulness of the social network. They were also asked for their Opinions on "skillemployment" compliance and knowledge of the skills required by the job market. As additional information, an interview guide administered to 24 graduates in employment situation. The results show that the use of the social network, in the integration process, does not guarantee the conformity or suitability of "skills-employment" among graduates on the labor market.
\end{abstract}


Keywords: Competence, employability, diploma, professional training

\section{Introduction}

En Côte d'ivoire tout comme en Afrique subsaharienne, les échecs relevés au niveau de l'insertion des diplômés, en général et ceux des ETFP, en particulier, s'expliquent en partie par l'inadéquation entre les qualifications des diplômés et les emplois sollicités. Cette étude investit les milieux de formation académiques professionnels privés pour faire ressortir les perceptions des diplômés du BTS, candidats à l'obtention d'un emploi au regard de leur capacité professionnelle. Pour ce faire, elle analyse les opinions des apprenants sur les résultats de la formation professionnelle pour tenter de répondre à la question suivante :

«Comment concilier adéquatement compétence professionnelle et emploi, chez les diplômés des ETFP ?»

Cette préoccupation est d'actualité puisqu'elle alimente le débat, en Côte d'ivoire, sur la conformité du binôme "compétences-employabilité » face aux spécificités actuelles du marché de l'emploi. La notion «employabilité » nous situe sur la capacité d'un individu à intéresser des employeurs et à développer durablement un projet professionnel. Cette définition empruntée à Guichard et Huteau (2007) commande de connaître le contenu des compétences inhérent à l'emploi, mais également, de renforcer ses capacités dans un cadre global d'ajustement des compétences, en raison de la dynamique évolutive du marché de l'emploi.

\section{I/CADRE THEORIQUE}

\section{I-1/ Etat du problème de recherche}

Selon l'OCDE (2011), les causes de l'inadéquation «qualificationemploi » sont plurielles et liés au fait que des travailleurs, de qualifications égales, présentent une grande hétérogénéité de compétences. En d'autres termes, à niveau d'éducation formelle égal, certains individus obtiennent des emplois exigeant un niveau de compétence élevé. Par contre, les autres obtiennent des emplois moins exigeant en termes de compétence. Les premiers donneront l'impression d'être qualifiés ou surqualifiés, puisqu'ils mettent en œuvre les compétences acquises en formation et des stratégies de capitalisation de compétence supplémentaires; tandis que les second, dont ce n'est pas le cas, se sentiront moins compétents. L'on observe alors une différence entre le niveau de scolarité atteint par l'employé et le niveau requis par l'emploi. Cela s'observe également avec des individus insérés dans des domaines d'activité inappropriés, eu égard à leur formation initiale. Ces derniers courent le risque de subir un certain déclassement, synonyme de sous-emploi ou d'échec dans le processus d'accession à l'emploi. 
Lorsqu'on regarde les statistiques nationales sur l'emploi, on observe que le taux de chômage est estimé à $9,6 \%$ chez les jeunes et à $17,1 \%$ chez les diplômés du supérieur (ENV, 2015). Ce qui nous indique clairement le nombre alarmant de chômeur en Côte d'ivoire. Le taux d'insertion des diplômés, issus de 56 établissements de l'ETFP pour l'année 2016, selon la Direction de l'Apprentissage et de l'Insertion Professionnelle (DAIP), est seulement de $14,43 \%$ pour 9.906 diplômés. Ce qui signifie que très peu de diplômés ont accès à l'emploi, contrairement à la forte majorité $(86 \%)$ qui devra développer d'autres réseaux d'insertion. Face à ces constats, on est en droit de s'interroger sur les difficultés que vivent les diplômés sur le marché de l'emploi certes, mais n'oublions pas, également, que le marché du travail est caractérisé par de forts taux de sous-emplois affectant la qualité des emplois. En effet, selon le rapport de l'ENSETE (2013), le sous-emploi visible lié au temps de travail affecte $22,4 \%$ des personnes actives (14-24 ans) et $23,8 \%$ des actifs ayant une formation de niveau supérieur (ENSETE, 2013). Par ailleurs, une étude menée auprès des entreprises ivoiriennes indique une prédominance de l'emploi informel dont la source serait liée à l'incapacité des pays en développement à transformer les activités en emploi ${ }^{1}$.

En Côte d'Ivoire, l'une des réactions institutionnelles à la faiblesse du taux d'insertion des diplômés et à leur maintien dans le milieu professionnel est l'orientation des activités de l'AGEFOP $^{2}$ vers la formation, l'orientation et l'insertion des diplômés. D'autres voies de succès entreprise par l'institutionnel visent à promouvoir une insertion harmonieuse des diplômés, notamment, par la mise en place de structure de placement au sein des établissements de formation.

Malgré ces efforts, les rapports d'évaluation, conduits par les chercheurs et experts, ont identifié un certain nombre de facteurs à l'origine de la faiblesse des résultats de l'institutionnel. Le plus congrue à cette étude, citée par Aska (2007), est la gestion structurelle et organisationnelle des établissements de formation professionnelle. Ces dernières devraient être à mesure d'adapter et d'actualiser les méthodes d'enseignement suivant les aspirations des entreprises pourvoyeuses d'emploi. Selon Kouakou et Koba (2015), le chômage des jeunes du milieu professionnel, notamment, des diplômés des grandes écoles privées (35,7\% pour le BTS en 2014) est dû au manque de qualification pratique au moment de la formation.

1 «Employabilité et Entrepreneuriat des jeunes en Côte d’Ivoire », Dr Kouakou. K. Clément, BIT. Page 51.

${ }^{2}$ L'Agence Nationale de la Formation Professionnelle (AGEFOP) est un Établissement Public à Caractère Industriel et Commercial (EPIC), créé par décret $\mathrm{N}^{\circ}$ 92-316 du 15 Mai 1992 et modifié par décret $N^{\circ} 2012-987$ du 10 octobre 2012. 
En marge du processus de formation, les trajectoires d'insertion, selon les travaux empiriques de Koné (2017), Akpe (2015) et N'Guessan (2015), relèvent l'ingérence prépondérante d'un réseau relationnel (parents, amis et connaissances), en raison de la faiblesse du dispositif institutionnel. En effet, Brou (2014), Koné et al. (2017) indiquent, également, que la question de l'insertion socioprofessionnelle des jeunes diplômés est associée, sous cet aspect, aux inégalités sociales ; car le diplômé qui a la capacité de mobiliser un fort capital social (relations politiques, administratives, culturelles, symboliques...) aura plus de chance à l'insertion professionnelle que celui qui n'en dispose pas ou faiblement.

Aux dires de ces chercheurs, les trajectoires d'insertion des diplômés sont définies par leur réseau d'appartenance relationnel. Lorsque le dispositif institutionnel d'insertion ne permet pas de juguler le nombre important de diplômés dans le marché de l'emploi, d'autres stratégies sont alors légitimées, en occurrence le capital social. Ceux qui en disposent sont voués à une insertion certaine, ceux en disposent moins, le sont relativement et ceux qui n'en disposent pas, non. Sous cet aspect, comment expliquer le fait que ce réseau relationnel n'opère pas de facto les meilleurs choix d'emploi qui concilient « formation initiale - compétence - emploi pour les diplômés qui en disposent?

L'objectif de cette étude est de montrer que le réseau social ou relationnel explique l'inadéquation (compétence-emploi) sur le marché de l'emploi.

La responsabilité du capital social, baptisé «réseau social» dans cette étude induit une hypothèse majeure qui est la suivante : Le recours au réseau social dans le processus d'insertion, est la cause de la non-conformité ou de l'inadéquation « compétence-emploi) rencontrées par les diplômés sur le marché de l'emploi.

\section{II/ Méthodologie \\ II-1/ Cadre géographique}

Prenant pour référence le classement de mérite opéré par le ministère de ministère de l'enseignement supérieur et de la recherche scientifique ${ }^{3}$ en 2011, nous avons admis d'entrée de jeu que les dysfonctionnements observés dans les meilleurs établissements sont au moins partagés par ceux aux bas du classement. Ainsi donc, notre investigation empirique s'est déroulée dans les trois meilleurs établissements supérieurs professionnels. Il s'agit des établissements :

$\begin{array}{ll}\text { - } & \text { LOKO, } \\ \text { - } & \text { PIGIER }\end{array}$

\footnotetext{
${ }^{3}$ http://www.enseignement.gouv.ci/index.php?open=actualite\&actu=class_ecole
} 
- et INPRAT,

Respectivement classées premier, second et troisième au niveau national.

\section{II-2/ Population et échantillonnage}

\section{Population}

La population concernée par cette étude est exclusivement constituée des étudiants diplômés du BTS (voir tabeau1) des filières industrielles et tertiaires issues des trois établissements ci-dessus citées.

TABLEAU 1 : Population Etudiants

\begin{tabular}{|l|c|c|c|c|c|}
\hline Etablissements & \multicolumn{2}{|c|}{$\begin{array}{c}\text { Population } \\
\text { totale } \\
\text { d'étudiants } \\
\text { diplômés } \\
\mathbf{2 0 1 2}\end{array}$} & \multicolumn{2}{|c|}{$\begin{array}{c}\text { Nombre d'étudiants par } \\
\text { filière pour l'année 2012 }\end{array}$} & $\begin{array}{c}\text { Pourcentage d'étudiant } \\
\text { par filière pour l'année } \\
\mathbf{2 0 1 2}\end{array}$ \\
\hline & & Industrielle & Tertiaire & Industrielle & Tertiaire \\
\hline Groupe LOKO & 1500 & 600 & 900 & 21,71 & 32,56 \\
\hline Groupe PIGIER & 929 & 0 & 929 & 0 & 33,61 \\
\hline INPRAT & 335 & 324 & 11 & 11,72 & 0,4 \\
\hline TOTAL & 2764 & 924 & 1840 & 33,43 & 66,57 \\
\hline
\end{tabular}

Source4 : données de thèse, Août-décembre 2012 (Akpe, 2015, pp.129)

\section{Echantillonnage}

La méthode des quotas a été adoptée pour sélectionner les individus. Fort de la représentativité, $1 / 10^{\mathrm{e}}$ de la population a été sélectionnée pour l'étude (voir tableau 2). Pour ce faire, une stratification multiple a été appliquée sur la population (voir tableau 1). Et au hasard de nos pérégrinations, nous avons pris soin de trouver nous-même les enquêtés.

TABLEAU 2 : Calcul de l'échantillon ${ }^{5}$

\begin{tabular}{|l|c|c|c|c|c|}
\hline \multirow{2}{*}{ ETABLISSEMENTS } & \multicolumn{2}{|c|}{$\begin{array}{c}\text { Pourcentage d'étudiant } \\
\text { par filière pour l'année } \\
\mathbf{2 0 1 2}\end{array}$} & $\begin{array}{c}\text { Echantillon }{ }^{7} \text { d'étudiant } \\
\text { diplômé par filière pour } \\
\text { l'année 2012 }\end{array}$ & TOTAL \\
\hline Groupe LOKO & Industrielle & Tertiaire & Industrielle & Tertiaire & \\
\hline Groupe PIGIER & 21,71 & 32,56 & 60 & 90 \\
\hline INPRAT & 0 & 33,61 & 0 & 93 & \\
\hline TOTAL & 11,72 & 0,4 & 32 & 1 & \\
\hline
\end{tabular}

Source : données de thèse, Août-décembre 2012 (Akpe, 2015, pp.129)

\section{II-3/Technique de collecte des données}

276 étudiants titulaires du BTS ont été interrogés par questionnaire. En guise de complément d'information, 24 diplômés BTS en emploi ont été interviewés à l'aide d'un guide d'entretien semi-structuré (24 diplômés en

4 Données d'enquête de terrain: AKPE, Y., H. (2015). "Qualité de la formation professionnelle dans les grandes écoles privées de Côte d'Ivoire ». Thèse unique, UFHB, Abidjan.

${ }^{5}$ Calcul de l'échantillon : $10 \%$ x 2764= 276,4 $\approx 276$

${ }^{7}$ Mode de calcul des échantillons par filière : \% étudiants par filière $\times$ échantillon 
emploi). Les données recueillies par questionnaire ont été statistiquement traitées, tandis que ceux issus du guide d'entretien, l'ont été grâce à analyse de contenus (qualitative). Les résultats sont présentés comme suit (voir résultats).

\section{III/ RESULTATS}

\section{III-/ Résultats d'enquête par questionnaire} figures.

Après enquête, les résultats obtenus ont été présenté à travers deux

Les résultats de la figure 1 montrent que sur 276 répondants :

- $\quad 87,13 \%$ sont "d'accord" avec la pensée suggérant que les connaissances à eux dispensées durant leur formation sont en cohérence avec les objectifs pédagogiques. Seulement 12,88\% disent ne pas être tout à fait d'accord contre $0,76 \%$ qui disent ne pas l'être du tout.

- $\quad 78,03$ \% sont « d'accord » qu'ils peuvent, grâce aux cours reçus, ils peuvent clairement établir un lien, une relation entre " théories » et «pratiques». Ce que n'admettent pas forcément $18,18 \%$ contre $3,79 \%$ qui ne l'admettent pas du tout.

- $\quad 85,71 \%$ disent avoir acquis des habiletés à l'issue de leur formation. 12,03\% disent ne pas être tout à fait d'accord avec cette acquisition d'habileté contre $2,26 \%$ qui disent ne pas l'être du tout.

- $\quad 86,26 \%$ disent que les habiletés acquises sont en conformité avec la demande du marché du travail. 9,16\% disent ne pas être vraiment d'accord contre 4,58\% qui disent ne pas l'être du tout.

- $\quad 83,33 \%$ sont d'accord pour dire qu'il est judicieux de recourir au réseau social dans le cadre de la recherche d'emploi. Ce qui n'est pas vraiment l'avis de $14,39 \%$ de répondants contre $2,27 \%$ qui disent ne pas être d'accord du tout.

En définitive, nous remarquons que la grande majorité des répondants a une bonne appréciation de la formation de BTS, reçu dans leurs établissements respectifs, et également de l'utilité du réseau social comme aide à la recherche d'emploi (voir figure 1). 


\section{FIGURE 1 : APPRÉCIATION DE LA FORMATION ET DE L'UTILITÉ DU RÉSEAU SOCIAL PAR LES RÉPONDANTS}

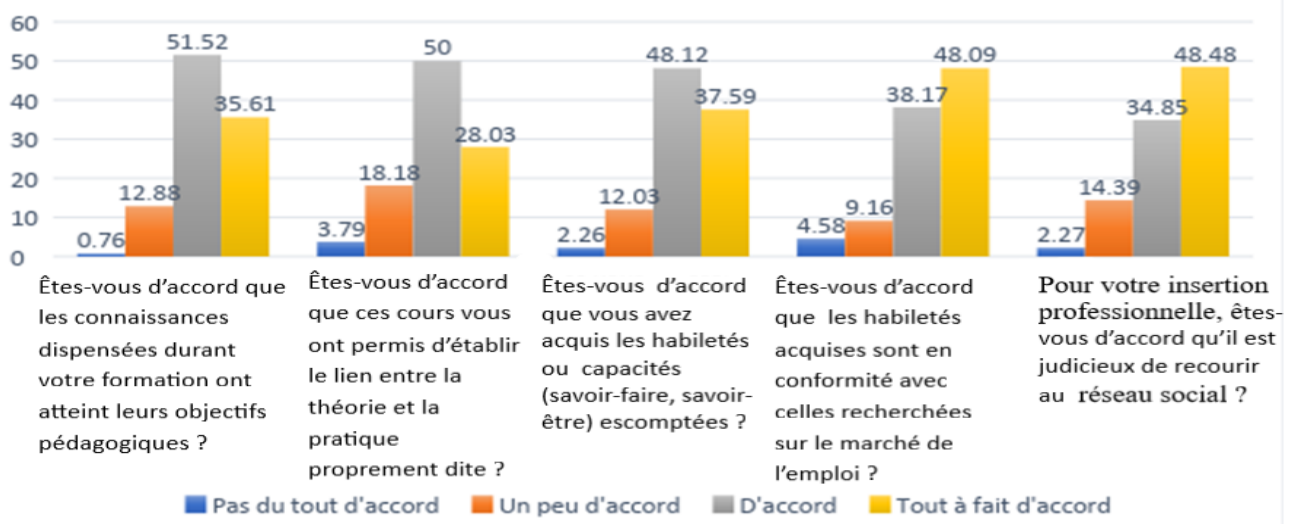

Source : données enquête de terrain, Août-décembre 2012

Les résultats de la figure 2 montrent que sur 276 répondants :

- $79,13 \%$ qui pensent que l'utilisation du réseau social ne garantit pas la conformité "compétence-emploi» chez les diplômés sur le marché du travail. Ce qui n'est pas l'avis des 20,87\% restant.

- Par ailleurs, sur la question concernant « les informations sur les compétences à développer dans les métiers exigés par la formation », les points de vue des répondants semblent s'équilibrer (49,12\% de non contre $50,88 \%$ de oui).

On retiendra de ces résultats que la majorité des répondants pense que l'utilisation du réseau social n'est pas un gage de conformité «compétence-emploi» chez les diplômés sur le marché du travail (voir figure 2). 
FIGURE 2: OPINIONS DES RÉPONDANTS CONCERNANT LA CONFORMITÉ « COMPÉTENCEEMPLOI » ET LES COMPÉTENCES EXIGÉES PAR LE MARCHÉ DE L'EMPLOI

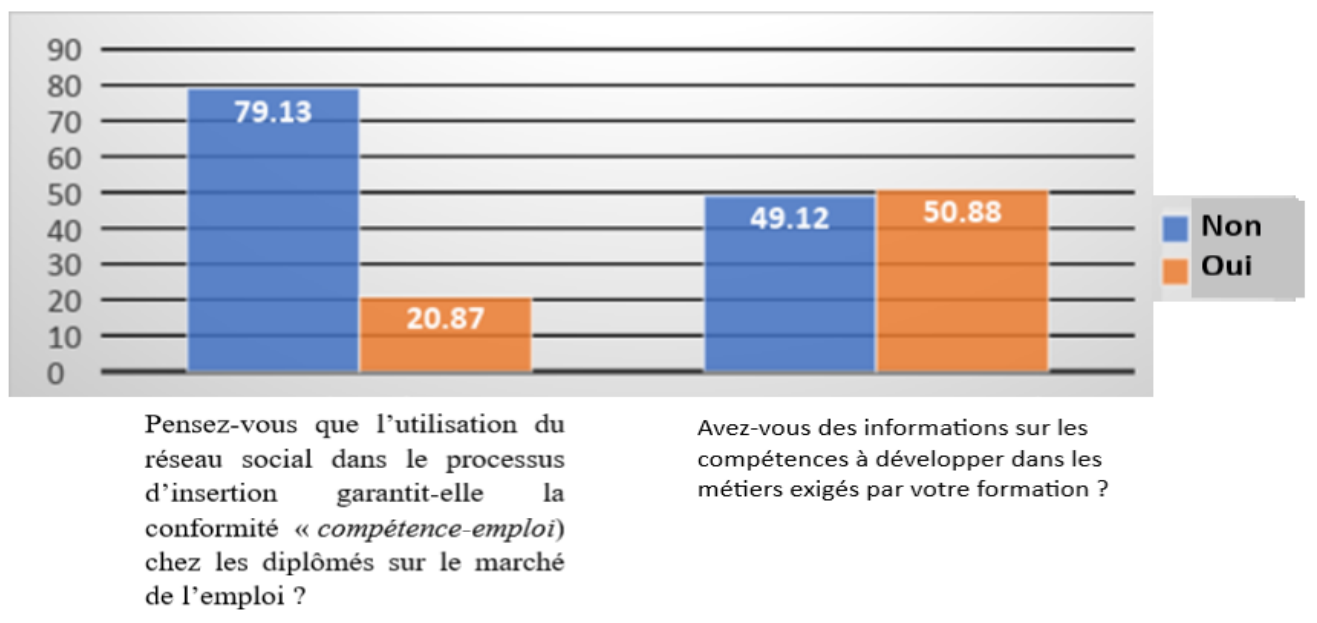

Source : données enquête de terrain, Août-décembre 2012

\section{III-2/ Résultats d'entretien (utilisation du guide d'entretien)}

Deux grandes catégories de diplômés se dessinent à l'issue du dépouillement des entretiens. On a, d'une part, les diplômés dont l'insertion professionnelle aura été guidée par l'établissement d'origine. Cette situation suppose que l'apprenant qui bénéficie d'un stage assisté par son établissement, devra au préalable être sélectionné parmi les meilleurs de sa cohorte. D'autre part, on a ceux qui ont construit leur insertion professionnelle en ayant recours à leur réseau social.

Pour la première catégorie d'étudiants, la présence d'une structure ou d'un cabinet de placement au sein de l'école est un atout dans le processus de placement de l'étudiant. Cette procédure à deux particularités. D'abord, elle rend efficace la formation en réduisant le temps d'inactivité de l'étudiant. Le temps d'inactivité étant celui qui s'écroule entre le moment de l'admission au BTS et l'insertion professionnelle.

Interrogée sur la question (temps d'inactivité) un diplômé argumente : "Moi, je n'ai pas duré, juste après le résultat du BTS, deux (2) jours après, j'ai commencé mon stage en entreprise. Donc depuis lors, je ne me suis plus assise, J'ai eu mon premier stage en septembre 2007... Quand j'ai fini mon premier stage, on peut dire que j'ai fait un (01) mois avant d'obtenir le deuxième et ainsi de suite... et j'ai eu mon emploi à partir du ler février 2009. " (Enquêté, étudiant en situation d'emploi).

Lorsque l'étudiant accumule de l'expérience dans plusieurs entreprises, il prend conscience de ses capacités et cherche par la même 
occasion une certaine efficience au niveau de sa rémunération. Quand ce n'est pas le cas, il tente de se perfectionner en vue d'une meilleure position sociale.

...J'ai fait le cycle ingénieur dans

une autre école pour avoir un

meilleur positionnement au niveau

de l'entreprise » (Enquêté, étudiant

en situation d'emploi) ...

Ensuite, la présence des structures de placement au sein des établissements permet une meilleure allocation des offres de stages, car ils sont étudiés en ce sens que la réponse de l'établissement et est plus adaptée. Cet état de fait est bien perçu par les étudiants à travers les réponses collectées sur les tâches à accomplir en entreprise.

... Toutes les tâches sont en rapport avec ma formation [...] Comme j'ai fait un BTS en assistanat de direction, cela me permet de pouvoir mieux recevoir les appels, rédiger les courriers, accueillir les clients et les employés [ ...] ce que je fais ici, je l'ai appris durant ma formation à PIGIER.... (Enquêté, étudiant en situation d'emploi).

Cette stratégie accorde à l'étudiant un meilleur suivi lors du stage et pendant la réalisation du rapport de fin d'études.

Pour la seconde catégorie d'étudiants, le processus d'insertion n'est pas à l'initiative de l'établissement d'origine. Il est le résultat d'une intervention du réseau social. Les raisons sont alors diverses. Les étudiants qui sont le plus souvent confrontés à cette situation sont ceux dont les structures de placement n'ont pas de solution d'insertion. Il y a aussi ceux dont l'établissement d'origine ne dispose pas de structure de placement. Enfin, il y a ceux qui optent pour un stage plus rémunéré que celui proposé par la structure de placement.

...J'ai déposé une demande et un beau matin, on m'a appelé pour le stage. Effectivement, il faut dire que j'ai ma petite sæur ici ... (Enquêté, étudiant en situation d'emploi)

Cette catégorie d'étudiants a la particularité d'éprouver beaucoup de difficultés ; puisque dans la réalité pour bon nombre d'entre eux, le métier embrassé ne correspond pas toujours exactement à la formation initiale. Ils semblent éprouver plus de mal à travailler en entreprise et estiment que la formation initiale est moins adaptée. 
...Il y a une grande différence, car la formation initiale est différente de la réalité pratique... (Enquêté, étudiant en situation d'emploi)

A la suite de cette seconde analyse, les entretiens menés montrent de façon générale que les étudiants de la première catégorie sont satisfaits de la formation reçue dans les grandes écoles privées, dans la mesure où ils n'éprouvent pas de réelles difficultés en entreprise. En cela, la politique d'insertion de l'établissement joue un rôle important dans l'accompagnement de l'étudiant dans sa nouvelle posture sociale. Cette politique, lorsqu'elle est bien coordonnée, à travers des actions menées par l'établissement, permet à l'étudiant d'être épanoui et de résister aux difficultés liées au stage et à l'emploi.

\section{IV/ DISCUSSIONS}

Cette étude trouve une explication, à travers ses résultats, à la préoccupation majeure liée à l'efficacité des pratiques d'insertion des diplômés des grandes écoles privées, partagés entre le recours au dispositif institutionnel et au réseau social. Les résultats indiquent que le recours au réseau social ou capital social semble être la voie la plus empruntée par les diplômés. Le capital social mobilisé dans le cadre de relations personnelles est légitimé par le diplômé comme ressource principal d'orientation sur le marché du travail. L'attitude du diplômé traduit le besoin d'atteindre des objectifs spécifiques, notamment l'accès à une position sociale de référence dans la société, mais également l'accès à des ressources sociales et à leur usage (Nan lin, 1995; Deschenaux, 2009). En outre, le recours au réseau relationnel garantit à priori l'accès à un emploi de sorte que, plus le réseau relationnel de l'individu est performant, plus vite il s'insère. Ce réseau est également le produit de stratégies d'investissement social, orientées vers la reproduction sociales directement utilisables par les diplômés des établissements supérieurs professionnels (Ponthieux, 2008).

L'étude montre également que les dispositifs institutionnels traduisent leur efficacité en matière d'insertion sociale. En effet, la politique institutionnelle d'insertion peut paraître insuffisante. Néanmoins, elle influence fortement la probabilité de réussite du diplômé, donc son employabilité. Cette thèse est susceptible d'orienter les politiques institutionnelles en ouvrant un cadre d'analyse des pratiques d'insertion formelles et la gestion des compétences individuelles (Ponthieux, 2008).

Dans cette perspective, la responsabilité des établissements de formation demeure l'une des conditions nécessaires à la mise en œuvre d'une telle réflexion. Cette responsabilité réside non plus dans la question de la qualité du diplômé, mais plutôt dans celle de l'information sur les 
perspectives professionnelles en lien avec la carrière sollicitée par l'apprenant.

Au cours de la formation, les étudiants estiment pouvoir atteindre les niveaux d'habiletés souhaitées. Ils estiment que la formation reçue leur permet d'avoir une idée des exigences du marché de l'emploi. En revanche, on note qu'environ un étudiant sur deux est susceptible de se prononcer sur les compétences réelles exigées pour l'emploi souhaité.

Le relevé des expériences, vécues au cours de la recherche d'emploi, indique bien que le dispositif institutionnel est très efficace pour soutenir l'employabilité du diplômé, car il assure une correspondance entre les qualifications professionnelles et les exigences en situation d'emploi. En effet, les résultats des pratiques d'insertion révèlent que les diplômés qui affirment être entré en stage par le biais de la structure de placement de leur établissement d'origine sont également ceux qui sont satisfait de l'emploi obtenu. Ils sont d'autant plus satisfaits du salaire obtenu qu'ils accusent le temps le plus court d'inactivité. Ce résultat est soutenu par les travaux de Kouadio (2007). Ce dernier indique que les difficultés d'insertion des diplômés de la formation professionnelle et technique sont liées à un déficit structurel et organisationnel. En revanche, l'appui du réseau social dans le processus de recherche d'emploi est l'une des voies prisées par les diplômés en raison du fait que les établissements offrent un nombre réduit de stage. Cette dernière peut s'avérer préjudiciable, car elle ne répond pas toujours à cette adéquation "compétences-employabilité ». Koné et al (2017) estiment que l'employabilité des jeunes passes inéluctablement par un suivi postformation dynamique et des dispositifs d'appui à l'insertion plus performants.

\section{CONCLUSION}

En somme, cette étude a situé le lien entre «compétence et employabilité »des diplômés des établissements de formations professionnelles privées de côte d'ivoire. Elle s'est consacrée à saisir les perceptions des diplômés des grandes écoles privées. Après avoir interrogé, par questionnaire, un échantillon de 276 apprenants BTS, sur 1'appréciation de la formation et de l'utilité du réseau social, sur la conformité « compétence-emploi » et les compétences exigées par le marché de l'emploi, et par entretien semi-structuré (guide d'entretien semi-ouvert) 24 diplômés BTS en emploi, sur leurs connaissances du marché de travail ; nous retiendrons que le recours au réseau social dans le processus d'insertion, ne garantit pas la conformité ou l'adéquation «compétenceemploi) chez les diplômés sur le marché de l'emploi. 


\section{References:}

1. Akpe, Y., H. (2015). Qualité de la formation professionnelle dans les grandes écoles privées de Côte d'Ivoire. Thèse unique, UFHB, Abidjan, $356 \mathrm{p}$.

2. Akpe, Y., H. (2015). Evaluation de la qualité de la formation dans les grandes écoles privées de Côte d'Ivoire ", Revue universitaire des Sciences de l'Education, EDUCI, Vol 2, pp. 98-118. ISSN 02585774

3. Brou, F. R. (2015). Problématique de l'insertion socioprofessionnelle des jeunes diplômés des universités publiques de Côte d'Ivoire : le cas de l'université Félix Houphouët Boigny. Thèse unique, UFHB, Abidjan.

4. Deschenaux, F., \& Laflamme, C. (2009). Réseau social et capital social: une distinction conceptuelle nécessaire illustrée à l'aide d'une enquête sur l'insertion professionnelle de jeunes Québécois. Sociologies.

5. Kouadio, A. (2007). L'enseignement général et l'enseignement technique et professionnel en Côte d'Ivoire: quelle articulation pour quels enjeux? Carrefours de l'éducation, (2), 217-232.

6. Guichard, J., \& Huteau, M. (2007). Orientation et insertion professionnelle : 75 Concepts clés. Dunod.

7. Kone, K. S., Koffi, K. A. et Ehui, P. J. (2016). Jeunes et insertion professionnelle en Côte d'Ivoire : La formation professionnelle à l'épreuve des pratiques d'insertion. Réseau Ouest Africain de Recherche en Education - ROCARE, Abidjan.

8. Lin, N. (1995). Les ressources sociales: une théorie du capital social. Revue française de sociologie, 685-704.

9. N'guessan, G., K., D. (2014). Le réseau social : un substitut à la régulation institutionnelle du marché du travail. Revue universitaire des Sciences de l'Education, EDUCI, Vol 2, pp. 98-118. ISSN 0258 5774.

10. Ponthieux, S. (2008). Rubrique - Les analyses du capital social : apports et controverses. Informations sociales, 147, (3), 32-33. https://www.cairn.info/revue-informations-sociales-2008-3.htmpage-32.htm. 